\title{
High-Energy Gamma-Ray Production in Microquasars
}

\author{
Hugo R. Christiansen, \\ Nucleo de Física, Universidade Estadual do Ceará, Av. Paranjana 1700, 60740-000, Fortaleza - CE, Brazil
}

Gustavo E. Romero, and Mariana Orellana

Instituto Argentino de Radioastronomía (IAR), C.C. 5, 1894 Villa Elisa, Argentina

Received on 19 January, 2007

\begin{abstract}
A hadronic model for gamma-ray production in microquasars is presented. Microquasars are galactic binary systems with jets, which have, presumably, hadronic components. We consider a microquasar formed by a neutron star that accretes matter from the equatorial wind of a Be primary star. The collision between the jet, emitted by the compact object, and the dense equatorial disk of the companion massive star is responsible for the gamma-ray production. Gamma-rays result from the decay of neutral pions produced in relativistic $p p$ interactions arising from this collision all along the orbit. Assuming a simple, positional independent set of parameters, our calculations are consistent with a peak of gamma-ray flux at the periastron passage with a secondary maximum near apastron. Under this assumption, gamma-ray signals would be in contrast with the radio/X-ray outbursts which peak clearly after periastron. We finally calculate the opacity of the ambient photon field to the propagation of the gamma-rays. The spectral energy distribution appears strongly attenuated in a wide band $(50 \mathrm{GeV}-50 \mathrm{TeV})$ due to local absorption. These spectral features should be detectable by an instrument like MAGIC through exposures integrated along several periastron passages.
\end{abstract}

Keywords: Galactic gamma-rays sources

\section{GENERAL REMARKS}

As we discuss in another work of these proceedings (see Christiansen (2006a) [1]) the study of high-energy gammarays and neutrinos is expected to provide primary hints about the origin of cosmic rays.

A suitable candidate for analyzing gamma-ray emission is the galactic microquasar LS I +61303 (LSI hereafter) for its particular features. It is indeed a well studied object whose parameters have been recently updated [2]. Furthermore, new generation instruments like MAGIC and GLAST are in a convenient position to detect gamma signals from this source. This situation allows making reliable predictions which could be contrasted in the near future.

LSI is a Be/X-ray binary system that presents a moderated $\mathrm{X}$-ray emission modulated with the radio emission period [3]. The system is composed by a compact objet (presumably a neutron star) and a Be (massive) primary. The X-ray/radio outbursts are triggered 2.5-4 days after the periastron passage of the compact object. A few years ago, Massi et al. [4] have detected the existence of relativistic radio jets in LSI extending up to about $400 \mathrm{AU}$ from the compact object [5].

LSI was originally associated with the COS-B gamma-ray source CG135+01, and later on with 3EG J0241+6103 [6, 7]. The gamma-ray signal is strongly variable and it has been recently shown that it peaks about periastron [8], in contrast to what happens with the radio/X-ray emission.

Although the matter content of microquasar jets is unknown, in the case of SS 433 iron X-ray line observations have proved the presence of ions in the jets $[9,10]$. We therefore assume that relativistic protons are present in LSI. The primary of the system generates a radially outflowing nearly equatorial wind. The compact object ( $\mathrm{CO}$ hereafter) moves around in a Keplerian orbit whose relative position is given

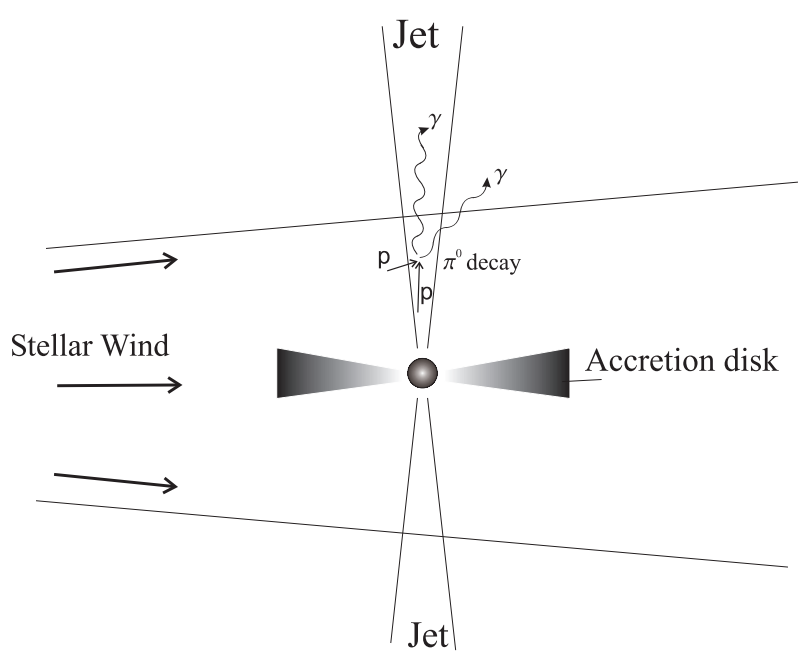

FIG. 1: A pictorical representation of high-energy gamma emission.

by $r(\psi)=a\left(1-e^{2}\right) /(1-e \cos (\psi))$. The (strong) radio signal peaks around $\psi=0.46 \pi$ [2]. The circumstellar disk has a half-opening angle $\phi=15^{\circ}$ with density $\rho_{\mathrm{w}}(r)=\rho_{0}\left(r / R_{*}\right)^{-n}$ where the wind velocity reads $v_{\mathrm{w}}=v_{0}\left(r / R_{*}\right)^{n-2}$ and $n=3.2$ $[11,12]$. The wind accretion rate onto the compact object is given by $\dot{M}_{\mathrm{c}}=4 \pi\left(G M_{\mathrm{c}}\right)^{2} \rho_{w}(r) / v_{\text {rel }}^{3}$ where $v_{\text {rel }}$ is its velocity relative to the circumstellar wind. The kinetic jet power $Q_{\mathrm{j}}$ is coupled to $\dot{M}_{\mathrm{c}}$ by $Q_{\mathrm{j}}=q_{\mathrm{j}} \dot{M}_{\mathrm{c}} c^{2}$ [13]. Only a small fraction of the jet particles are highly relativistic hadrons $\left(q_{\mathrm{j}} \sim 10^{-3}-10^{-2}\right)$ for most of the jet power consist of other particles. In particular, cold protons are ejected with a macroscopic Lorentz factor $\Gamma \sim 1.25$ [4]. The jet is a cone with a radius $R_{\mathrm{j}}(z)=z R_{0} / z_{0}$ and the jet axis, $z$, is taken normal to the orbital plane $[14,15]$. See Figure 1 to see a picture of the situation. 
TABLE I: Basic parameters assumed for the model

\begin{tabular}{lll}
\hline Parameter & Symbol Value \\
\hline Mass of the compact object & $M_{\mathrm{c}}$ & $1.4 M_{\odot}$ \\
Jet's injection point & $z_{0}$ & $10^{7} \mathrm{~cm}$ \\
Initial radius & $R_{0}$ & $z_{0} / 10$ \\
Mass of the companion star & $M_{\star}$ & $10 M_{\odot}$ \\
Radius of the companion star & $R_{\star}$ & $10 R_{\odot}$ \\
Effective temperature of the star & $T_{\mathrm{eff}}$ & $22500 \mathrm{~K}$ \\
Density of the wind at the base & $\rho_{0}$ & $10^{-11} \mathrm{~g} \mathrm{~cm}^{-3}$ \\
Initial wind velocity & $v_{0}$ & $5 \mathrm{~km} \mathrm{~s}^{-1}$ \\
Eccentricity & $e$ & 0.72 \\
Orbital period & $P$ & $26.496 \mathrm{~d}$ \\
\hline
\end{tabular}

As the $\mathrm{CO}$ moves around the primary, part of the bipolar jet crosses the circumstellar disk allowing high-energy interactions between the relativistic hadrons in the (normal) jet and the slow (radial) hadrons of the wind. For the relativistic proton flux, we use a power-law which in the lab (observer) frame reads [16])

$$
J_{p}\left(E_{p}, \theta\right)=\frac{c K_{0}}{4 \pi}\left(\frac{z_{0}}{z}\right)^{2} \frac{\Gamma^{-\alpha+1}\left(E_{p}-\beta_{\mathrm{b}} \sqrt{E_{p}^{2}-m_{p}^{2} c^{4}} \cos \theta\right)^{-\alpha}}{\sqrt{\sin ^{2} \theta+\Gamma^{2}\left(\cos \theta-\frac{\beta_{\mathrm{b}} E_{p}}{\sqrt{E_{p}^{2}-m_{p}^{2} c^{4}}}\right)^{2}}} .
$$

The angle subtended by the proton velocity direction and the jet axis will be roughly the same as that of the emerging photon which is detected $\left(\theta \approx \theta_{\mathrm{obs}}\right) ; \beta_{\mathrm{b}}$ is the bulk velocity in units of $c$, and $\alpha=2.2$ is in order to match the GeV gammaray spectrum observed by EGRET [17]. The normalization constant $K_{0}$ is related to the number density of particles $n_{0}{ }^{\prime}$ flowing in the jet at $R_{0}$ (see [15] for details). When the particle gyro-radius is smaller than the radius of the jet, the matter from the wind can penetrate the jet diffusing into it. We will treat the problem in a simple phenomenological way by means of a constant penetration factor $f_{\mathrm{p}} \sim 0.1$, which is in order to reproduce the observed gamma-ray flux at $\mathrm{GeV}$ energies, where opacity effects due to pair creation are unimportant.

\section{GAMMA RAY LUMINOSITY AND OPACITY}

Neutral pion decay following high energy proton collisions is a natural channel for (in jet) high energy gamma-ray production. The differential gamma-ray emissivity from $\pi^{0}$ decays can be expressed as [18]

$$
q_{\gamma}\left(E_{\gamma}, \theta\right)=4 \pi \eta_{\mathrm{A}} \sigma_{p p}\left(E_{\gamma}\right) \frac{2 Z_{p \rightarrow \pi^{0}}^{(\alpha)}}{\alpha} J_{p}\left(E_{\gamma}, \theta\right),
$$

(in $\mathrm{ph} \mathrm{s}^{-1} \mathrm{sr}^{-1} \mathrm{erg}^{-1}$ ), where $Z_{p \rightarrow \pi^{0}}^{(\alpha)}=0.092$ is the so-called spectrum-weighted moment of the inclusive cross-section and it is related to the fraction of kinetic proton energy transferred to the pions [19]. The parameter $\eta_{\mathrm{A}} \sim 1.4$ takes into account the contribution from different nuclei in the wind, and $\sigma_{p p}\left(E_{\gamma}\right) \approx 30\left[0.95+0.06 \log \left[E_{p}\left(E_{\gamma}\right) / \mathrm{GeV}\right]\right]$ (mb) [18] is the cross section for inelastic $p p$ interactions, for $E_{\gamma} \geq 1$
$\mathrm{GeV}$. For $\theta$ we will adopt a viewing angle $\theta_{\mathrm{obs}}=30^{\circ}$ in accordance with [2].

The spectral gamma ray intensity (in $\mathrm{ph} \mathrm{s}^{-1} \mathrm{erg}^{-1}$ ) is

$$
I_{\gamma}\left(E_{\gamma}, \theta\right)=\int_{V} d^{3} \vec{r} n(\vec{r}) q_{\gamma}\left(E_{\gamma}, \theta\right)
$$

where $V$ is the interaction volume between the jet and the circumstellar disk. The particle density of the wind that penetrates the jet is $n(r) \approx f_{\mathrm{p}} \rho_{w}(r) / m_{p}$, and the generated luminosity in a given energy band $\left[E_{\gamma}^{a}, E_{\gamma}^{b}\right]$ results

$$
L_{T}\left(E_{\gamma}^{a, b}, \theta\right)=\int_{E_{\gamma}^{a}}^{E_{\gamma}^{b}} d E_{\gamma} E_{\gamma} I_{\gamma}\left(E_{\gamma}, \theta\right)
$$

In order to predict the total gamma ray luminosity measurable on Earth, we must evaluate this integral above a threshold of detection which shall depend on the telescope characteristics. On the other hand, we should keep in mind that $E_{\gamma}$ cannot exceed the maximum gamma ray energy available from the hadronic processes described so far. When purely hadronic collisions are considered, pion production becomes the natural production channel. Thus, for $p p$ interactions, it is related to the maximal proton energy by $E_{p}^{\max }=6 E_{\gamma}^{\max }$ if we do not take into account the pion multiplicity. The minimum is fixed by the threshold for pion production $E_{p}^{\min }=$ $\Gamma\left[\left(2 m_{p}+m_{\pi}\right)^{2}-2 m_{p}^{2}\right] / 2 m_{p}$.

Relativistic protons in the jet will interact with target protons in the wind through the reaction channel $p+p \rightarrow p+p+$ $\xi_{\pi^{0}} \pi^{0}+\xi_{\pi^{ \pm}}\left(\pi^{+}+\pi^{-}\right)$where $\xi$ 's are the different multipicities. Isospin symmetry, which is in agreement with Fermi's original theory of pion production relates the three multiplicities with an equal value, thus we can use $\xi_{\pi}$ for all. According to [20], for inelastic $p p$ interactions the relation between the gamma-ray energy and the proton energy is modified as

$$
E_{p} \approx 6 \xi_{\pi}\left(E_{p}\right) E_{\gamma} / K
$$

The inelasticity coefficient is $K \sim 0.5$ since on average a leading nucleon and a pion cloud both leave the interaction fireball each carrying half of the total incident energy. For the energy dependent pion multiplicity we will follow the prescription adopted by Mannheim and Schlickeiser (1994) [21] $\xi_{\pi} \simeq\left(E_{p} / \mathrm{GeV}-1.22\right)^{1 / 4}$, which should be valid up to $10^{4}$ $\mathrm{GeV}$, and adopt a softer root growing law for higher energies (see Christiansen et al. (2006b) for details [27]).

Under these assumptions, it can be shown that the maximum proton energy produced amounts to more than $50 \mathrm{PeV}$ at periastron and lies below $15 \mathrm{PeV}$ near apastron $[1,27]$. It can be derived from the simple argument that the Larmor radius of the particle should be smaller than the size of the acceleration region $[22,23]$. Since energy losses inside LSI can be neglected, we can write $E_{p}^{\max }=R(z) \mathrm{e} B(z)$., where $R$ is the jet radius. Assuming that both matter and field follow adiabatic evolution when moving along the jet, we can assume equipartition between the magnetic field energy and the kinetic energy of the jet. Then, the magnetic field in the jet reference frame reads $[24,25] B(z, \psi)=4 G M_{\mathrm{c}} R(z)^{-1} \sqrt{\pi q_{\mathrm{j}} \rho_{w} v_{\mathrm{j}} / v_{\mathrm{rel}}^{3}}$, 
where $v_{\mathrm{j}}=c \beta_{\mathrm{b}}$ is the jet cold proton velocity and we correspondingly adopt $q_{\mathrm{j}} \sim 0.1$.

As a result of the discussion above, the maximal gamma energies of $p p$ origin rise up to $170(40) \mathrm{TeV}$ at periastron (apastron). We can therefore predict the gamma ray total luminosity in the whole $\left[E_{p}^{\min }, E_{p}^{\max }(\psi)\right]$ energy band. For the source LSI, our result amounts to $5.310^{34}\left(10^{35}\right) \mathrm{erg} \mathrm{s}^{-1}$, depending on whether we adopt $q_{j}=10^{-3}\left(10^{-2}\right)$ for the relativistic protons.

Now, there is in fact an experimental constraint on the luminosity, as recently analyzed by Fegan et al. (2005). In [26], it is claimed that above $0.35 \mathrm{TeV}$ the total gamma ray flux must satisfy

$$
\frac{1}{4 \pi d^{2}} \int_{0.35 \mathrm{TeV}}^{\infty} d E_{\gamma} I_{\gamma}\left(E_{\gamma}\right) \leq 1.710^{-11} \mathrm{ph} \mathrm{cm}^{-2} \mathrm{~s}^{-1} .
$$

Assuming that we can produce ultra high energy with the same model, our calculations give about four times this value, so we need to show that most of the high-energy gamma rays get absorbed. Infrared photon fields can be responsible for HE photon absorption at the source. To show it, we calculate the optical depth $\tau$ within the circumstellar disk for a photon with energy $E_{\gamma}$ and $E_{\mathrm{ph}}$ for the ambient photons,

$$
\tau\left(l, E_{\gamma}\right)=\int_{E_{\min }^{e e}}^{\infty} d E_{\mathrm{ph}} \int_{l}^{\infty} d l n_{\mathrm{ph}}\left(E_{\mathrm{ph}}, l\right) \sigma_{e^{-} e^{+}}\left(E_{\mathrm{ph}}, E_{\gamma}\right) .
$$

$\sigma_{e^{-} e^{+}}\left(E_{\mathrm{ph}}, E_{\gamma}\right)=\frac{\pi r_{0}^{2}}{2}\left(1-\xi^{2}\right)\left[2 \xi\left(\xi^{2}-2\right)+\left(3-\xi^{4}\right) \ln \left(\frac{1+\xi}{1-\xi}\right)\right]$ is the photon-photon pair creation cross section, where $r_{0}$ is the classical radius of the electron and $\xi=\sqrt{1-\frac{\left(m_{e} c^{2}\right)^{2}}{E_{\mathrm{ph}} E_{\gamma}}}$. $n_{\mathrm{ph}}\left(E_{\mathrm{ph}}, l\right)$ is their density at a distance $l$ from the neutron star, and $E_{\mathrm{min}}^{e e}=m_{e}^{2} c^{4} / E_{\gamma}$ is the threshold energy for pair creation in the ambient photon field. This field can be considered as formed by two components, one from the Be star and another from the cold circumstellar disk. Here,

$$
n_{\mathrm{ph}, 1}\left(E_{\mathrm{ph}}, l\right)=\left(\frac{\pi B\left(E_{\mathrm{ph}}\right)}{h c E_{\mathrm{ph}}}\right) \frac{R_{\star}^{2}}{l^{2}+r^{2}-2 l r \sin \theta},
$$

is for the black body emission from the star, and

$$
n_{\mathrm{ph}, 2}\left(E_{\mathrm{ph}}, l\right)=\left(\frac{\pi B\left(E_{\mathrm{ph}}\right)}{h c E_{\mathrm{ph}}}\right) \frac{r^{2}}{l^{2}},
$$

corresponds to the emission of the circumstellar disk. In both cases we adopt

$$
B\left(E_{\mathrm{ph}}\right)=\frac{2 E_{\mathrm{ph}}^{3}}{(h c)^{2}\left(e^{E_{\mathrm{ph}} / k T_{\mathrm{eff}}}-1\right)}
$$

where $T_{\text {eff }, 1}=22500 \mathrm{~K}$ and $T_{\text {eff, } 2}=17500 \mathrm{~K}$ [11]. Since the value for $T_{\mathrm{eff}, 2}$ is only valid in the inner region of the disk, we adopt $r=r_{\text {periastr. }}$. In any case, computing the opacity at periastron, where the luminosity is maximal, will be enough for our purposes of proving that the total gamma-ray luminosity predicted in our model is below the Fegan et al.'s constraint

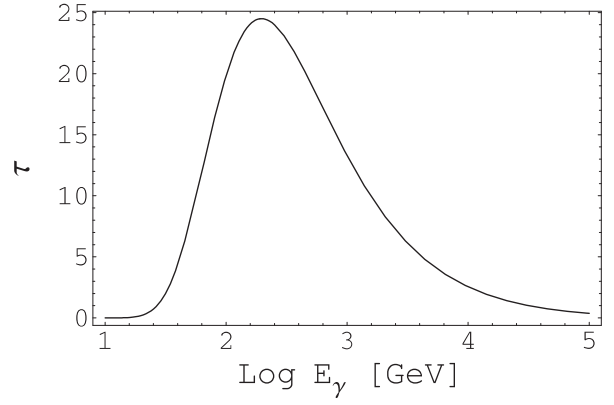

FIG. 2: Optical depth at periastron, as a function of $\log \left(E_{\gamma} / \mathrm{GeV}\right)$ (see Ref.[27]).

[26]. Figure 2 shows that the optical depth remains above unity for a wide range of photon energies particularly about $E_{\gamma} \approx 250 \mathrm{GeV}$ where it presents a sharp knee. As a result, the opacity-corrected total flux above $E_{\gamma}=350 \mathrm{GeV}$ drops from $7.110^{-11}$ down to $1.410^{-12} \mathrm{ph} \mathrm{cm}^{-2} \mathrm{~s}^{-1}$, lying well below the Fegan et al.'s threshold.

Low energy observing windows, however, could promptly reveal the presence of a hadronic gamma-ray source at the position of LSI. In particular, between 1 and $50 \mathrm{GeV}$ the opacity is sufficiently low as to allow a relatively easy detection by instruments like the ground-based MAGIC telescope and the LAT instrument of GLAST satellite. LS I +61303 is an outstanding candidate to corroborate that high-energy emission is a common property of microquasars. A neutrino detection from this source would be also a major achievement, which would finally solve the old question on whether relativistic protons are part of the matter content of the energetic outflows presented by accreting compact objects (see Christiansen et al. (2006b) for further discussion [27]).

We have also calculated the spectral luminosity $L_{s}$ in an energy region of central interest. For this, we used an approximate expression

$$
L_{S}\left(E_{\gamma}, \theta\right)=E_{\gamma}^{2} I_{\gamma}\left(E_{\gamma}, \theta\right) \exp \left(-10 E_{\gamma} / E_{p}^{M}\right)
$$

where $E_{p}^{M}=100 \mathrm{TeV}$ (see Fig. 3 and Ref.[28] for details). Note that the minimum does not occur exactly at apastron. This is due to the particular orbital variation of the accretion rate. Furthermore, there is a second maximum, related to the function $1 / v_{\text {rel }}^{3}$, which grows dramatically when the compact object gets far from the primary star and moves more parallel to the weak stellar wind.

\section{CONCLUSIONS}

We presented a model for gamma-ray production in microquasars assuming pure hadronic interactions. Our approach is rather complementary to leptonic models which might dominate at lower gamma-ray energies and after the periastron passage. Our model was based on the interaction of a polar jet above a neutron star, with the dense equatorial disk of the companion massive star. Gamma-rays are the result of 

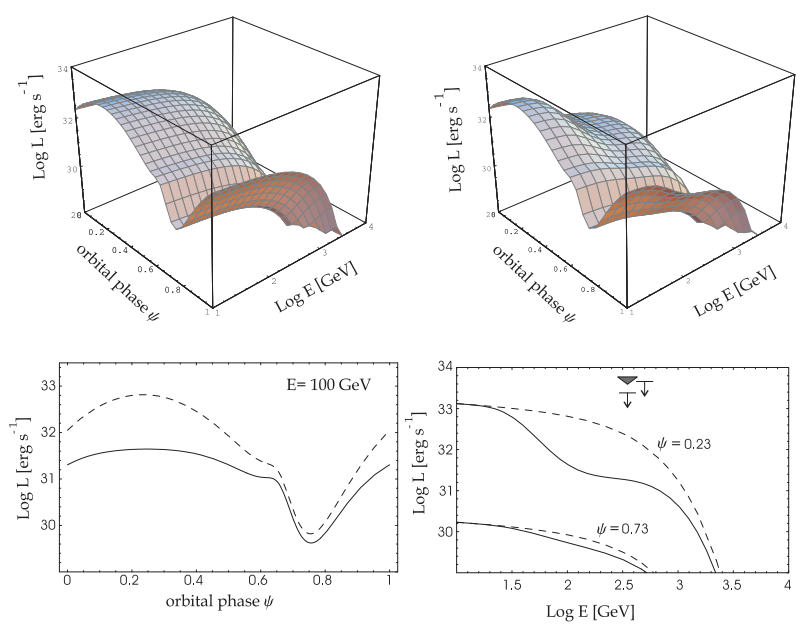

FIG. 3: Upper, Left: A three-dimensional plot shows the spectral luminosity as a function of the orbital phase and gamma-ray energy. Right: The same plot, taking into account the gamma-ray absorption in the ambient photon fields. Down, Left: Ligthcurve for gammarays of energy $100 \mathrm{GeV}$. The dashed curve corresponds to the generated spectral luminosity, whereas the continuous curve takes into account the effects of photospheric opacity. Right: Spectral energy distribution at the periastron and apastron passage. The unabsorbed spectra are in dashed lines. Upper limits from Whipple observations are indicated. For these plots we adopted a conservative $q_{j}=10^{-3}$ value (see Ref.[28]). the decay of neutral pions produced in relativistic $p p$ collisions. Assuming constant parameters for the system, our predictions are consistent with a peak of gamma-ray emission at the periastron passage, with a secondary maximum at phase $\psi \sim 4 / 3 \pi$ when the accretion rate has also a local maximum i.e. when the wind velocity is more parallel to the relative velocity. This is in contrast with the radio/X-ray outbursts which peak after the periastron. We finally take into account the opacity of the ambient photon fields to the propagation of the gamma-rays. The spectral features should be detectable by an instrument like MAGIC and GLAST. Charged pion decay also leads to neutrino production as discussed in another work of the present meeting [1] (see also [27]).

After the completion of this paper, the MAGIC telescope found, indeed, evidence of variable high-energy gamma ray emission at a few hundred $\mathrm{GeV}$ [29]. At the light of these newest results, some parameters can be adjusted in order to improve the hadronic model 's predictions. For instance, a velocity dependent penetration factor could correctly shape the observed gamma-ray flux with its precise time dependent features (see [30]).

Acknowledgements H.R. Christiansen is grateful to Fundação de Amparo à Pesquisa do Ceará for full support of the expenses related to this meeting. This work has been supported by CNPq and FUNCAP, Brazil, and CONICET and ANPCyP, Argentina.
[1] H.R. Christiansen, Braz. J. Phys. 37, 642 (2007).

[2] J. Casares, I. Ribas, J.M. Paredes, et al., MNRAS 360, 1105 (2005).

[3] J.M. Paredes, J. Martí, M. Peracaula, and M. Ribó, Astron. and Astrophys. 320, L25 (1997).

[4] M. Massi, M. Ribó, J.M. Paredes, et al., Astron. and Astrophys. 376, 217 (2001).

[5] M. Massi, M. Ribó, J.M. Paredes, et al., Astron. and Astrophys. 414, L1 (2004)

[6] P.C. Gregory and A.R. Taylor, Nature 272, 704 (1978).

[7] D.A. Kniffen, W.C.K. Alberts, D.L. Berstch, et al. Astrophys. J. 486, 126 (1997).

[8] M. Massi, Astron. and Astrophys. 422, 267 (2004).

[9] T. Kotani, N. Kawai, M. Matsuoka, and W. Brinkmann, PASJ 48, 619 (1996).

[10] S. Migliari, R. Fender, and M. Méndez, Science 297, 1673 (2002).

[11] J. Marti and J.M. Paredes, Astron. and Astrophys. 298, 151 (1995).

[12] P.C. Gregory and C. Neish, Astrophys. J. 580, 1133 (2002).

[13] H. Falcke and P. L. Biermann, Astron. and Astrophys. 293, 665 (1995).

[14] V. Bosch-Ramon, G.E. Romero, and J.M. Paredes, Astron. and Astrophys. 429, 267 (2005).

[15] G.E. Romero, D.F. Torres, M.M. Kaufman Bernadó, and F. Mirabel, Astron. and Astrophys. 410, L1 (2003).
[16] D. Purmohammad and J. Samimi, Astron. and Astrophys. 371, 61 (2001).

[17] R.C. Hartman, D.L. Bertsch, S.D. Bloom, et al., Astrophys. J. S. S. 123, 79 (1999)

[18] F. A. Aharonian and A. M. Atoyan, Astron. and Astrophys. 309, 917 (1996).

[19] T.K. Gaisser, Cosmic Rays and Particle Physics, Cambridge University Press, Cambridge 1990.

[20] V.L. Ginzburg and S.I. Syrovatskii, Sov. Astron. 8, 342 (1964).

[21] K. Mannheim and R. Schilckeiser, Astron. and Astrophys. 286, 983 (1994).

[22] A. M. Hillas, ARAA 22, 425 (1984).

[23] W. Bednarek, G. F. Burgio, and T. Montaruli, New. Astron. Rev. 49, 1 (2005).

[24] R.D. Blandford and A. K onigl, Astrophys. J. 232, 34 (1979).

[25] V. Bosch-Ramon, G.E. Romero, and J.M. Paredes, Astron. and Astrophys. 447, 263 (2006).

[26] S. Fegan, et al., Astrophys. J. 624, 638 (2005).

[27] H.R. Christiansen, M. Orellana, and G.E. Romero, Phys. Rev. D 73, 063012 (2006); H.R. Christiansen, Phys. Scripta 127, 82 (2006).

[28] G.E. Romero, H.R. Christiansen, M. Orellana, Astrophys. J. 632, 1093 (2005).

[29] J. Albert, et al., Science 312, 1771 (2006).

[30] M. Orellana, G.E. Romero, astro-ph/0608707. 